\title{
Endoscopic ultrasound assessment of gastrointestinal polypoid lesions of indeterminate morphology in patients with portal hypertension
}

\section{(ㄷ)(우우}

\author{
Authors \\ Dimitrios E. Sigounas, Amanullah Shams, Peter C. Hayes, John N. Plevris
}

Institution

Centre for Liver \& Digestive Disorders, Royal Infirmary of Edinburgh, Edinburgh, United Kingdom

submitted 17.4.2017

accepted after revision $\quad 25.10 .2017$

Bibliography

DOI https://doi.org/10.1055/s-0043-124363 |

Endoscopy International Open 2018; 06: E292-E299

(c) Georg Thieme Verlag KG Stuttgart · New York

ISSN 2364-3722

Corresponding author

Professor John Plevris, PI, Hepatology Laboratory, Associated PI CIR, University of Edinburgh, First Floor Chancellor's Building, 49 Little France Crescent, Edinburgh EH16 4SA

j.plevris@ed.ac.uk

\section{ABSTRACT}

Background and study aims Polypoid lesions found during upper gastrointestinal endoscopy (UGIE) are occasionally found in patients with portal hypertension $(\mathrm{PH})$. This study aimed to assess the true nature of such polypoid lesions using endoscopic ultrasound (EUS) and determine the accuracy of UGIE in differentiating between vascular and non-vascular lesions in $\mathrm{PH}$.

Patients and methods We retrospectively assessed all patients with PH referred for EUS due to polypoid lesions of unknown nature at UGIE over a 7-year period. Cases of known varices were excluded. UGIE findings were compared to EUS findings.

Results 66 patients were included (26 male). Commonest UGIE findings were: possible varices (19.4\%), polypoid/neoplastic lesion (52.8\%) and submucosal lesion (16.7\%). After EUS, the final diagnoses were: varices in $25 \%$, polypoid lesion with underlying vessel/varix in $27.8 \%$ and non-vascular lesion or submucosal lesion in $47.2 \%$. The diagnostic accuracy of UGIE was suboptimal, since $28.6 \%$ of possible varices were eventually found to be non-vascular, while $15.8 \%$ of polyp/neoplastic looking lesions proved to be varices and $42.1 \%$ were lesions with underlying vessel/varix. $50 \%$ of submucosal lesions were eventually found to be varices. Conclusion Endoscopists should have a high index of suspicion of varices or polyps related to varices when assessing atypical looking polypoid lesions in patients with $\mathrm{PH}$. In such cases EUS should be considered before obtaining biopsies.

\section{Introduction}

Portal hypertension $(\mathrm{PH})$ is associated with well-known gastrointestinal mucosal abnormalities such as portal hypertensive gastropathy (PHG), enteropathy and colopathy, as well as varices and gastric antral vascular ectasias (GAVE) [1 - 3].

Polypoid lesions can also be found in this context, but the significance of such lesions has not been thoroughly appreciated. Some may represent actual adenomatous or hyperplastic polyps, while some may represent or be associated with varices with atypical morphology. The nature of those has not been systematically assessed. In recent years, a distinct type of polypoid lesions in the context of $\mathrm{PH}$ has also been reported in both pediatric [4] and adult population [5]. Location of these lesions varies in different reports, but are more commonly seen in the duodenum, small bowel [6-8] and stomach [5,9-13]. There is no consensus on the terminology used to describe such lesions and various terms including "gastric polyposis associated with portal hypertension" [11] or "portal hypertension-associated gastric polyps" [9] have been proposed. More recently the term "Portal hypertensive polyp (PHP)" has been suggested $[12,14]$ and this will be used in this report.

There are no standardized criteria for the diagnosis of PHP. Their appearance is quite variable: they can be sessile or pedunculated, single or multiple, measuring from 3 to $30 \mathrm{~mm}[5,12]$. A commonly seen endoscopic feature is a whitish exudate overlying the polyp [5]. Gastric antrum and duodenum seem to be the most common PHP sites [5,12]. Histological features described include polypoidal configuration of the mucosa, lamina propria edema, proliferating submucosal thick-walled capillar- 
ies, foveolar hyperplasia and minimal or absent inflammation $[5,7,11,12]$. The exact histological description varies between different reports, but it is clear that these polyps exhibit features distinguishing them from other types of polyps [12]. PHP can be associated with active bleeding, melaena and anemia and are frequently found during relevant investigations [5]. The exact prevalence of those lesions is not well defined and most studies published so far are case reports or case series. 2 recent cohort studies cite a prevalence of 0.9 to $1.4 \%[5,12]$ in patients with cirrhosis and portal hypertension having an esophagogastroduodenoscopy (EGD). There are no data regarding the prevalence of PHP in the small and large intestine.

The identification of this PH-related entity has generated some further questions. Firstly, it is unclear how close PHP are related to other endoscopic manifestations of $\mathrm{PH}$. Particularly in view of the polypoidal appearance of these lesions, it is important to know if they are related in any way to the presence of varices or underlying vessels, as this could potentially make polypectomies or even simple biopsies hazardous. Furthermore, it is debatable whether it is possible to accurately identify $\mathrm{PHP}$ and differentiate them from other $\mathrm{PH}$ related lesions, in particular varices or other non-PH related pathology, using standard upper $\mathrm{Gl}$ endoscopy alone. This raises the question whether the use of complementary modalities such as endoscopic ultrasound (EUS) would be required to rule out the presence of varices or highly vascular lesions in such polypoid lesions.

This study aimed to investigate the nature of polypoid lesions seen in the upper gastrointestinal (GI) tract in the context of portal hypertension using EUS. Furthermore, we assessed the ability of diagnostic endoscopy to differentiate between vascular and non-vascular lesions in $\mathrm{PH}$, using EUS diagnosis as reference.

\section{Patients and methods}

This was a retrospective, single-center study, performed in a tertiary liver center in Scotland, UK. All patients with PH referred for EUS due to polypoid lesions of uncertain nature seen in EGD between June 2008 and November 2015 were included. In all cases, the referring endoscopist was interested in ruling out the possibility of a vascular polypoid lesion or underlying varices, but the degree of confidence regarding the true nature of the lesions varied. In all cases the Olympus UE260 electronic radial echoendoscope was used.

Data on etiology of $\mathrm{PH}$, severity of cirrhosis (if present), Child-Pugh score, Model for End-stage Liver Disease (MELD) score, platelet count, location of the lesion(s) in question, endoscopic findings related to $\mathrm{PH}$ (esophageal and gastric/ectopic varices, PHG), pathology results when available and EUS findings were all retrieved from the hospital's endoscopy database and electronic case records.

By definition, all patients referred for EUS had $\mathrm{PH}$ and in all cases there was uncertainty regarding the nature and the vascularity of the lesion(s) in question: in particular, whether they represented varices with atypical appearance when seen at initial endoscopy. Cases of obvious esophageal or gastric/ectopic varices referred for EUS confirmation or assessment following eradication therapy were excluded. Even though image enhancing modalities like narrow band imaging (NBI) had been used in some endoscopies, the diagnostic confidence in the context of portal hypertensive gastropathy was generally low, therefore our analysis did not specifically assess whether such techniques were used and in how many patients. Only the final endoscopy outcome was used, regardless of the exact modalities implemented in each case.

To facilitate analysis of the findings at the initial endoscopy reports, the lesions were categorized as follows: 1 . possible varix; 2. polypoid/neoplastic-looking lesion; 3. possible submucosal lesion; 4. possible extrinsic compression; 5.Ulceration; 6. prominent folds. This was done retrospectively, based on the referring endoscopist's description of the lesion(s) in question. The categories were formed retrospectively after reviewing all the available descriptions in the endoscopy reports. Therefore, if the lesion was described as polyp or possible cancer, this would be categorized in group 2, whereas if the lesion was described as benign looking ulcer it would be categorized in group 5 . The authors did not make any assessments of their own for 2 reasons. Firstly, because the photos taken during an endoscopy cannot be a reliable substitute for the actual endoscopy experience and secondly because the intention was to capture the average endoscopists' ability to accurately assess the nature of polypoid lesions in the context of $\mathrm{PH}$ rather than expert endoscopists in endoscopy of the liver patient. The referring endoscopists came from different medical and health allied professions including general Gastroenterologists, Surgeons and Nurse-endoscopist and from a mix of teaching and district general hospitals.

Similarly, EUS findings were categorized as follows: 1. Varices; 2 . polyps with underlying vascular structure/varix; 3 . nonvascular polyp; 4. extrinsic compression by another structure; 5. Ulcer; 6. submucosal lesion; and, finally 7. normal appearances under EUS. Varices were defined as a network of mucosal or submucosal hypoechoic structures with positive EUS Doppler signal. Polyps with underlying vessel were defined as mucosal hyperechoic homogeneous lesions sitting on a network of hypoechoic structures with positive EUS Doppler signal and a feeding vessel to the polyp arising from the network of vessels. Non-vascular polyps were defined as mucosal hyperechoic lesions without visible coexisting vascular network and a negative EUS Doppler signal. Cases where a definite extrinsic structure was seen causing the endoscopic abnormality (e.g. the gallbladder), were categorized in category 4 . When only an ulcer was seen without any vascular component, it was categorized in category 5 and when a lesion exhibited ultrasonographic characteristics compatible with submucosal lesions, it was included in category 6. Category 7 comprised the few cases where no abnormality was detected by EUS.In all cases, care was given to differentiate vascular dilatations from varices. Vascular dilations tend to be sporadic and non-communicating while varices were recognized as a network of vessels penetrating the muscularis with significant tortuosity.

All cases were subsequently categorized in 3 groups by considering all clinical information available. These included endo- 
scopic, pathology and EUS findings. The 3 categories were: 1. Varices; 2. polypoid lesion with underlying vessel/varix; and 3. non-vascular lesion (including normal appearances). Vascular lesions (categories 1 and 2) were either certainly (varices) or potentially related to $\mathrm{PH}$ (category 2 ), whereas non-vascular lesions (category 3 ) were less likely to be associated with $\mathrm{PH}$.

Such categorization felt to be clinically relevant and important as performing a biopsy or polypectomy of a polypoid lesion that is an unrecognized varix can lead to life-threatening bleeding. Therefore, the aforementioned categorization can be informative both in assessing the association of the lesions in question with $\mathrm{PH}$, as well as in order to evaluate the safety of potential endoscopic interventions.

\section{Ethics}

This study was conducted in accordance with the United Kingdom (UK) research ethics guidelines. After review by the local ethics committee, further specific ethical review and approval were not required, as the study was considered a retrospective clinical audit work, using data already obtained as a part of regular patient care.

\section{Statistical methods}

Only descriptive statistics were used. No formal statistical comparisons were made. Data are presented as frequencies for categorical data and as mean with the associated $95 \%$ confidence intervals $(95 \% \mathrm{Cl})$ or median with the relevant interquartile range (IQR) for continuous variables. SPSS 21.0 (IBM corp., Version 21.0, Armonk, NY, USA) was used for all analyses.

\section{Results}

\section{Patient characteristics}

A total of 36 patients were included. Patient's characteristics, endoscopy findings, pathology results and final diagnosis are presented in $>$ Table 1 . The majority of patients were male with a 2.6/1 ratio and mean age was 67.7 years. Alcoholic liver disease (ALD) and non-alcoholic fatty liver disease (NAFLD) were the 2 main causes of $\mathrm{PH}$ accounting for more than $80 \%$ of cases with small predominance of ALD. Only 1 patient had $\mathrm{PH}$ related to portal vein (PV) thrombosis. Median MELD score was 9. Two-thirds of patients belonged to Child-Pugh class A. Only $9.1 \%$ belonged to class $C$. 1 patient had pre-hepatic portal hypertension and therefore Child-Pugh score was not calculated, while 2 patients were referred from other hospitals just for the EUS procedure and there were not enough data in the records available to calculate the score.

\section{Endoscopic findings}

Half of the lesions seen were found in the distal part of the stomach (Incisura, antrum and prepyloric area) and $19.4 \%$ were seen in the duodenum. Another quarter of lesions were found in proximal stomach. There were endoscopic findings of portal hypertension present in endoscopy in the vast majority of cases (94.4\%). Esophageal varices were the most common finding (72.2\%), followed by PHG and gastric or duodenal varices, both seen in slightly less than half of patients. The appear-
- Table 1 Epidemiological characteristics, endoscopy findings and pathology results for 36 patients referred for further assessment with EUS following an initial EGD, due to suspicion that polypoid or submucosal appearing lesions might be vascular in the context of portal hypertension. Those lesions were not considered as definite varices, but the degree of confidence about their nature varied.
Male, $\mathrm{n}(\%)$
$26(72.2)$

Age, Mean $(95 \% \mathrm{Cl})$

$67.7(64.5-70.8)$

PH aetiology

- ALD $16(44.4)$

- NAFLD

$14(38.9)$

- HCV $2(5.6)$

\begin{tabular}{ll} 
- PBC & $1(2.8)$ \\
\hline
\end{tabular}

- Cryptogenic 2(5.6)

- PV/SV thrombosis $1(2.8)$

MELD score, median (IQR) $\quad 9(7-12)$

\begin{tabular}{l} 
PLT number, mean $(95 \% \mathrm{Cl}) \quad 145.5(126.5-164.5)$ \\
\hline
\end{tabular}

Child-Pugh class, $\mathrm{n}(\%)$

\begin{tabular}{|c|c|}
\hline - A & $22(66.7)$ \\
\hline - B & $8(24.2)$ \\
\hline - C & $3(9.1)$ \\
\hline
\end{tabular}

- $\mathrm{C}$

$3(9.1)$

Location of lesion, $\mathrm{n}(\%)$

- Esophagus 4(11.1)

- Proximal stomach-EG] 5 (13.9)

- Gastric body $5(13.9)$

- Incisura, antrum, prepyloric $18(50)$

- Duodenum 7 (19.4)

Evidence of PH in endoscopy 34 (94.4)

$\begin{array}{ll}\text { Esophageal varices, } \mathrm{n}(\%) & 26(72.2)\end{array}$

$\begin{array}{ll}\text { PHG, } n(\%) & 17(47.2)\end{array}$

Gastric/ectopic duodenal varices, $\mathrm{n}(\%)$

Pathology, n (\%)

- No samples taken $19(52.8)$

- Inflammatory/hyperplastic/granulation/ 8(22.2) ulceration

- Chemical gastropathy

$1(2.8)$

- Adenoma

$3(8.3)$

- Cancer $2(5.6)$

- Normal

ALD, alcoholic liver disease; CI, confidence interval; EUS, endoscopic ultrasound; MELD, model for end - stage liver disease; NAFLD, non-alcoholic fatty liver disease; EGD, esophagogastroduodenoscopy; EG], esophago-gastric junction; PH, portal hypertension; PLT, platelets; PV, portal vein; SV, splenic vein 

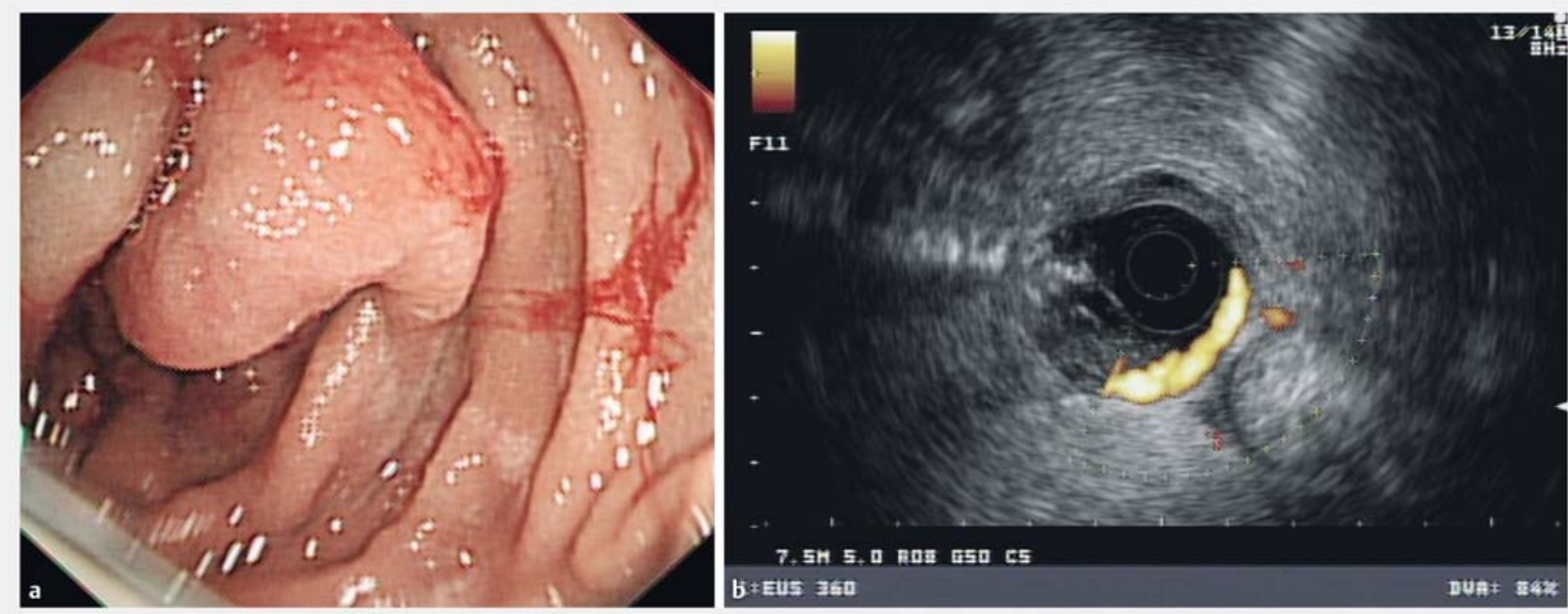

- Fig. 1 a Duodenal polypoid lesion biopsied previously leading to bleeding. Endoscopic view revealing portal hypertensive enteropathy and a polypoid lesion with some visible contact bleeding. b Duodenal polypoid lesion biopsied previously leading to bleeding. Under EUS, varices in the second and third parts of the duodenum.
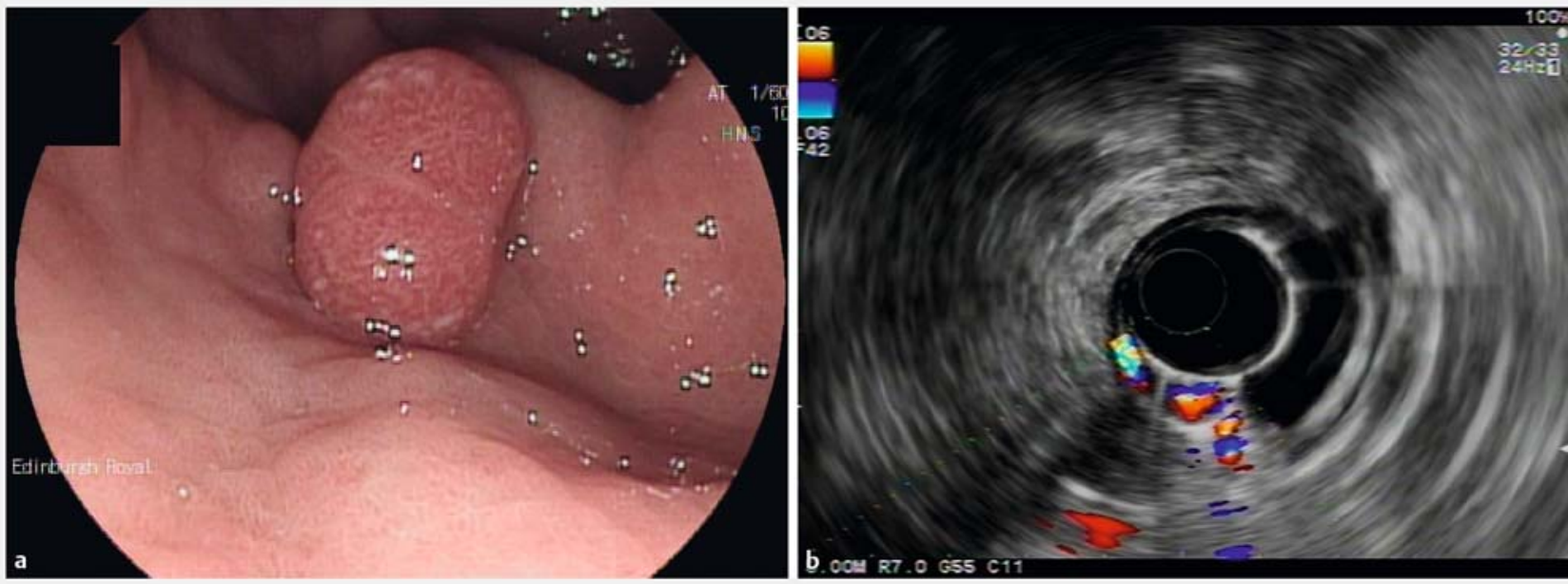

- Fig. 2 a Polypoid lesion in antrum; Endoscopic view of portal hypertensive gastropathy and antral polyp. b Polypoid lesion in antrum. Under EUS, appearance of pseudopolyp formation over ectopic gastric varices in antrum.

ance of these polypoid lesions varied significantly and some representative examples of the endoscopic appearance followed with the EUS findings are presented in > Fig. 1, > Fig. 2, $\triangleright$ Fig. 3, $>$ Fig. 4 , and $>$ Fig. 5.

In half the cases, no biopsies were taken. Of the cases where samples were obtained, nearly half showed inflammatory or hyperplastic changes or were compatible with granulation or ulceration. 3 patients had biopsies confirming adenomas and 2 had cancer diagnosed. The decision to biopsy was made by each individual endoscopist based on the findings on initial endoscopy or after EUS was performed. There were no standard criteria used as this was a retrospective assessment.
Initial endoscopic impression was categorized as possible varix in $19.4 \%$ of cases, while in more than half the white light endoscopic appearances were more suggestive of a neoplastic/ polypoid lesion. Submucosal lesion was considered as the most likely diagnosis in $16.7 \%$ of cases with the rest of categories (possible extrinsic compression, ulceration and prominent folds) accounting for approximately $10 \%$ of the total cases.

\section{Endoscopic ultrasound appearances}

EUS findings were compatible with varices (occluded or not) in 10/36 patients and an equal number of cases (10/36) were found to have polyps with underlying vessels. 8 patients $(8 / 36)$ had non-vascular polyps. Interestingly, 3 patients had a normal 

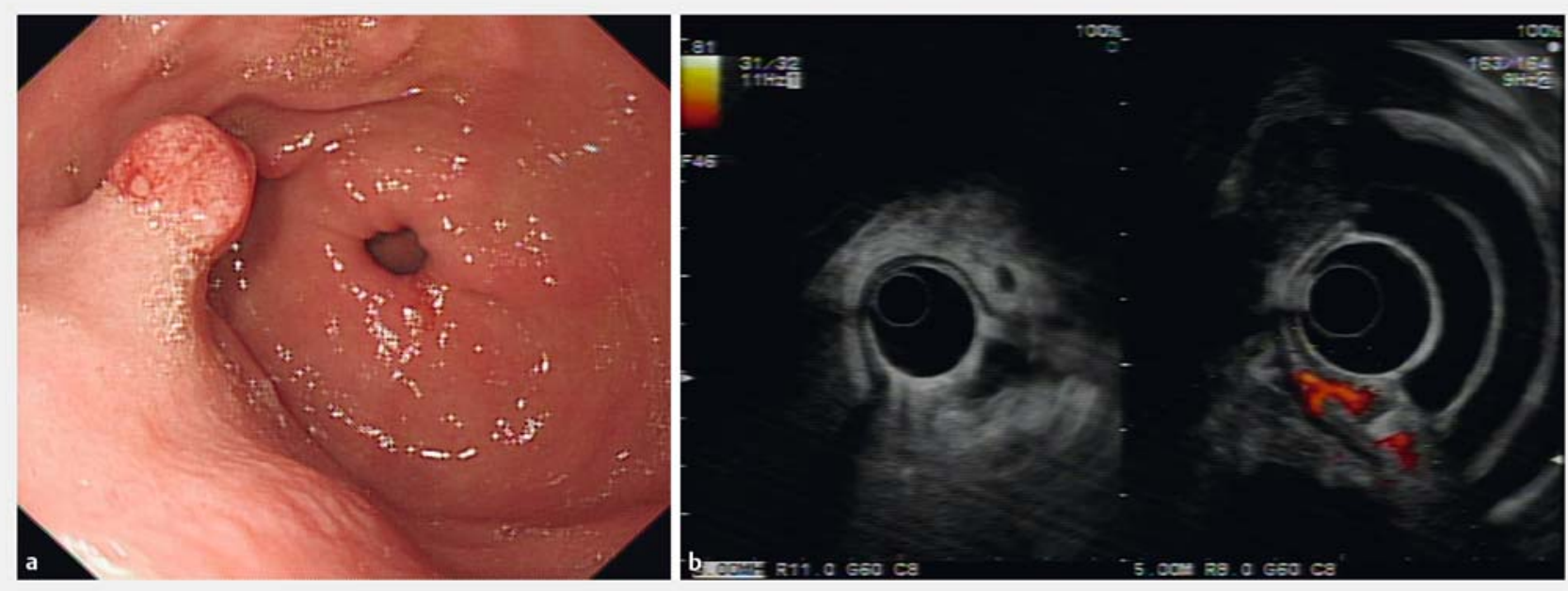

- Fig. 3 a Prepyloric polypoid lesion; Endoscopic view of prepyloric polyp. b Prepyloric polypoid lesion. Under EUS, appearances suggesting hyperplastic polyp with a feeding vessel, most likely representing a varix.
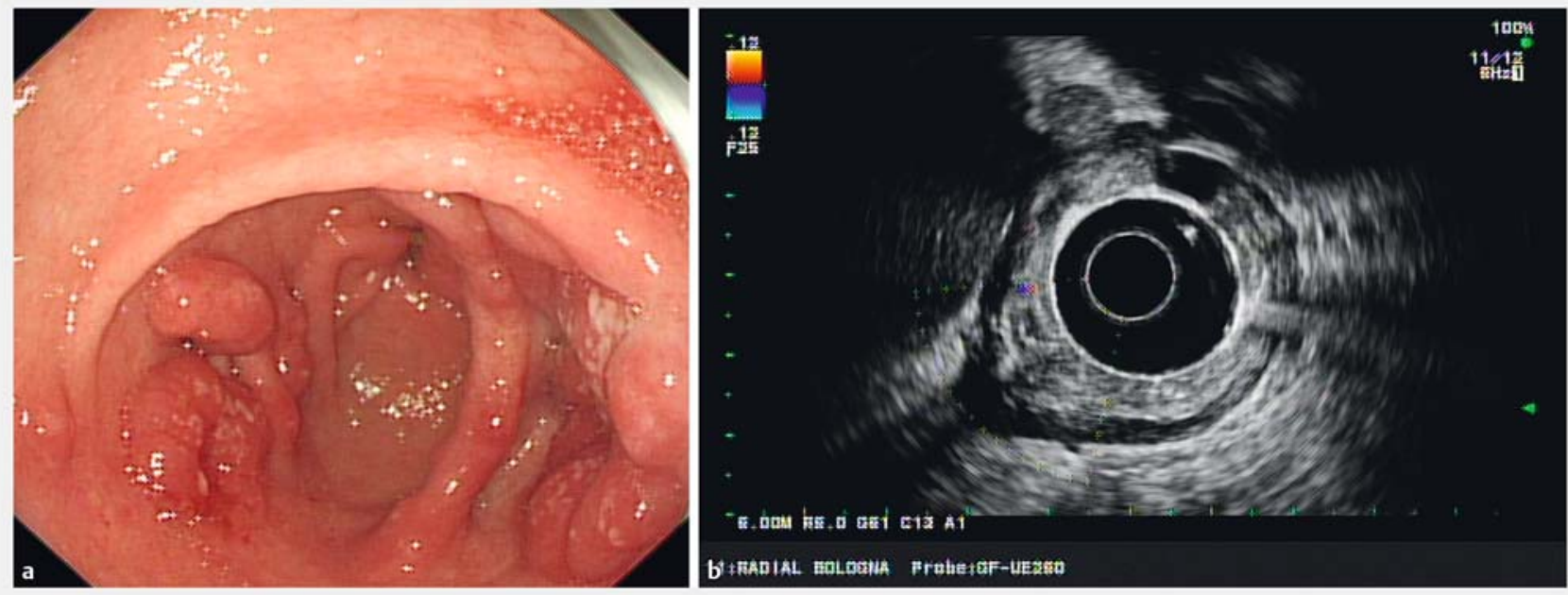

- Fig. 4 a Polypoid lesions in antrum; Endoscopic view of antral polypoid lesions. b Polypoid lesions in antrum. Under EUS, no blood vessels seen. Biopsies taken confirming inflammatory/hyperplastic polyp.

EUS. 2 cases of submucosal lesions and 2 of extrinsic compression were documented. Diagnosis was ulcer in 1 patient.

Final EUS diagnosis was non-vascular lesions or normal in almost half of the patients (47.2\%) while the rest was equally divided between varices and polypoid lesions with underlying varices/vascular structures.

Comparing the initial endoscopic impression with the final diagnosis after EUS and pathology where available, showed that more than a quarter of vascular/variceal looking lesions proved to be non-vascular. Conversely, amongst the lesions categorized as neoplastic/polypoid in the initial endoscopy, almost $60 \%$ proved to be vascular and, in fact, $15.8 \%$ were varices. Of the 6 submucosal-appearing lesions, 3 proved to be varices. Extrinsic compression, ulceration and prominent folds (total of 3) seen in initial endoscopy were confirmed as non-vascular after EUS. The relevant results are presented in $\mathbf{D}$ Table $\mathbf{2}$.

\section{Discussion}

This study utilized EUS to assess in more detail upper gastrointestinal polypoid lesions of uncertain nature, in patients with $\mathrm{PH}$. This revealed that a significant number of those lesions that did not have the typical appearance of varices in OGD, were indeed varices or were associated with underlying vascular structures in more than half of cases. When comparing the initial endoscopic impression with the actual outcome of investigations when EUS results were available, it was evident that 6 out of 9 patients with documented varices seen in EUS, were referred as possible polyps or submucosal lesions. Furthermore, 

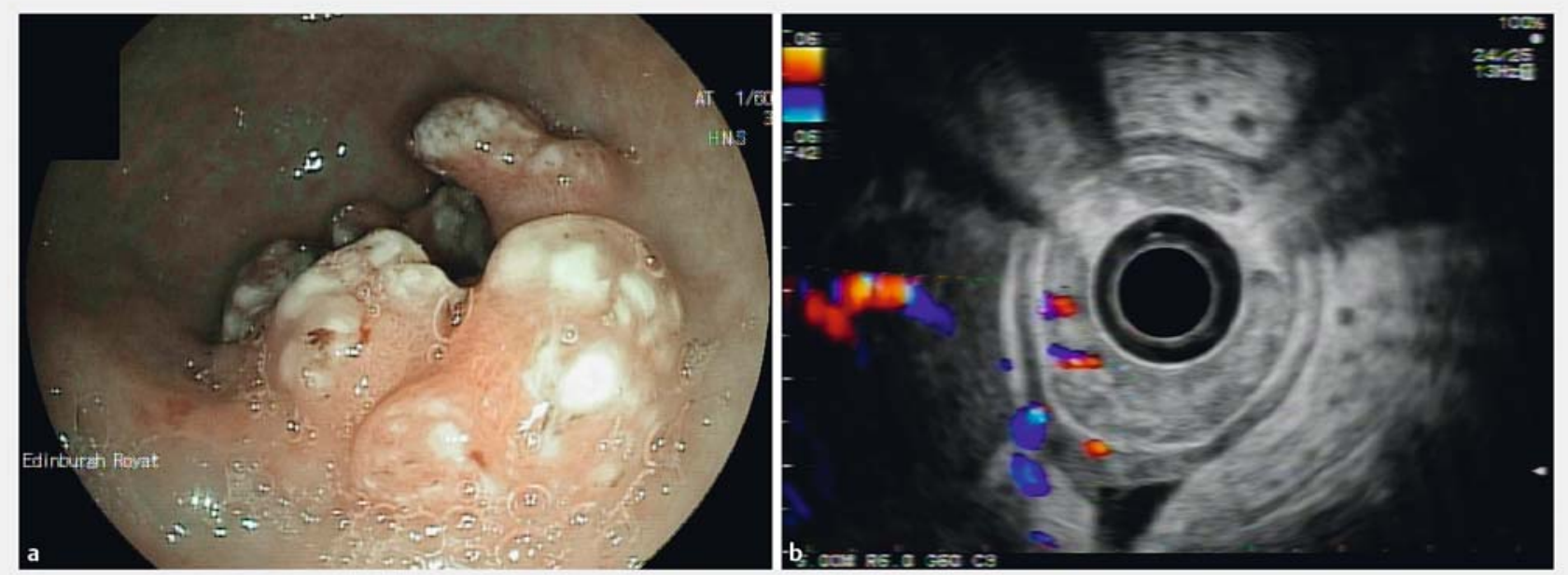

Fig. 5 a Patient originally investigated due to iron deficiency anaemia. Extensive prepyloric polypoid lesion found, extending into the pyloric channel; Endoscopic impression of ectopic gastric varices. Patient had thrombin injection. b Patient originally investigated due to iron deficiency anaemia. Extensive prepyloric polypoid lesion found, extending into the pyloric channel. Under EUS, increased vasculature with evidence of previous thrombin injection.

- Table 2 Correlation between endoscopic impression as documented at index endoscopy and the final diagnosis after EUS + /- pathology results were available for 36 patients without definite/known varices at diagnostic endoscopy. Percentages of EUS final diagnoses are given for each category of endoscopic diagnoses.

\begin{tabular}{|c|c|c|c|c|c|}
\hline & & \multicolumn{3}{|c|}{ Final categorization after EUS \pm pathology } & \multirow[t]{2}{*}{ Total } \\
\hline & & Vascular/varices & $\begin{array}{l}\text { Polypoid with under- } \\
\text { lying vessel/varix }\end{array}$ & Other non-vascular & \\
\hline \multirow{6}{*}{$\begin{array}{l}\text { Endoscopic } \\
\text { findings }\end{array}$} & Possible varix, n (\%) & $3(42.9)$ & $2(28.6 \%)$ & $2(28.6 \%)$ & $7(100)$ \\
\hline & Polypoid/neoplastic, n (\%) & $3(15.8)$ & $8(42.1)$ & $8(42.1)$ & $19(100)$ \\
\hline & Submucosal, n (\%) & $3(50)$ & $0(0)$ & $3(50)$ & $6(100)$ \\
\hline & Possible extrinsic compression, $\mathrm{n}(\%)$ & $0(0)$ & $0(0)$ & $1(100)$ & $1(100)$ \\
\hline & Ulceration, n (\%) & $0(0)$ & $0(0)$ & $1(100)$ & $1(100)$ \\
\hline & Prominent fold(s), n (\%) & $0(0)$ & $0(0)$ & $2(100)$ & $2(100)$ \\
\hline \multicolumn{2}{|l|}{ Total } & $9(25)$ & $10(27.8)$ & $17(47.2)$ & $36(100)$ \\
\hline
\end{tabular}

EUS, endoscopic ultrasound

$42.1 \%$ of cases referred as polyps, turned out to be related to varices, having a prominent vascular component, while $15.8 \%$ proved to be varices. Even though the neoplastic/polypoid lesions comprised $52.8 \%$ of subjects in this study, it is this type of lesions that is more likely to be biopsied. In almost $60 \%$ of these lesions there was a significant vascular component including varices, and this needs to be kept in mind when dealing with such lesions. These results highlight the importance of detailed assessment of polypoid lesions in the context of $\mathrm{PH}$ and endoscopists should have a high index of suspicion of being varices or vascular polyps associated with underlined varices. In addition to this, endoscopic impression may be misleading and the decision to take biopsies from such lesions of uncertain appearance should be carefully and thoroughly weighted against the risk of bleeding.
In the authors' experience, the use of image enhancing techniques, such as narrow band imaging (NBI), to differentiate polypoid lesions related with portal hypertension or varices from adenomatous or dysplastic polyps is not as informative as the presence of background portal hypertensive gastropathy is associated with abnormal vascular mucosal patterns, thus making the interpretation difficult. In addition, the literature regarding the usefulness of image enhancing techniques in the context of $\mathrm{PH}$, is lacking. It would be of importance to study whether NBI and similar techniques could help differentiate dysplastic from vascular lesions thus potentially averting the need for biopsy or EUS. The use of image enhancing techniques was not consistently documented in the endoscopy reports, even though such technique is routinely used in our centre and most likely used in most cases. However, since this was a 
retrospective study and there is little evidence of the value of such technique in $\mathrm{PH}$, we chose not to include such information in this study.

Even in the absence of overt varices increased vasculature within the wall of the stomach and oesophagus due to collateral veins related with portal hypertension is often associated with the development of hyperplastic polyps. Such polyps developing over a network of collaterals that is often detected by endoscopic ultrasound may have an increased risk of bleed even if they don't not lie on varices.

Even though studies on PHP so far have not demonstrated an increased risk of clinically significant bleeding following biopsies or polypectomies $[5,10]$, it is important to recognize that most studies are retrospective and comprise no more than 14 patients, while many are just case-reports and case-series. In fact, 3 of 14 patients in the study by Lemmers et.al [5] presented with active bleeding, even though the actual lesions were not considered to be the source of bleeding. It is unknown if any of the polypoid lesions in the Lammers et al. series were varices, but this seems unlikely. It is relatively reassuring in that study, that none of the 12 patients that had biopsy, polypectomy or mucosectomy had any significant bleeding, and the presence of underlying vessels on EUS was not associated with increased risk of bleeding.

It is noteworthy that 1 patient in our series was referred from another hospital due to severe variceal bleeding following biopsies from a polypoid lesion in the duodenum to exclude malignancy. Bleeding was successfully treated with thrombin injection, but this case highlights that severe bleeding remains a possibility. Even if the overall risk is relatively low $(5.8 \%$ of the biopsies in our series), this risk should be balanced against the potential benefits on an individual basis and should be attempted only if the Endoscopy unit staff have the necessary means and experience to manage bleeding post biopsy in a liver patient. For certain patients with advanced liver disease, the risk of bleeding may be unacceptable compared with the potential benefit of biopsying or resecting a duodenal or gastric polypoid lesion. This is even more risky in the subgroup of patients with varices having a polypoid appearance ( $15.8 \%$ of the polypoid/ neoplastic lesions in this study). Such patients would have a very high risk of severe bleeding if polypectomy was to be attempted.

Whether our patient population is comparable to the patients included in previous reports on PHPs is unclear, since pathology was not obtained in a significant number of patients in our case. Similarities observed in the population distributions (predominantly male, mean age 67.7, mostly Child-Pugh class A) between our patients and patients in previous reports [5, 10], as well as the distribution of the lesions seen is suggestive, but not confirmatory that at least some of those lesions seen in our series were indeed PHPs. Pathology has been reported as hyperplastic, inflammatory, granulation or ulceration in 8 patients and it is possible that some other polyps not biopsied were also PHPs. Of those 8 patients, lesions in 3 were found to be a varix and 4 were found to overly a varix/penetrating vein. Interestingly none bled from the biopsy, but it is unclear what would have happened if a polypectomy had been attempted.
Whether the same risk applies to the lesions found to have increased vascularity particularly those overlying a varix, is difficult to assess, but this possibility needs to be kept in mind, until further studies answer this question.

This study has certain strengths. This is to our knowledge the only EUS assessment of upper gastrointestinal polypoid lesions in patients with $\mathrm{PH}$. The implementation of EUS enabled a more in-depth assessment of the complex vascular patterns encountered in the mucosa of patients with $\mathrm{PH}$. This revealed a previously unrecognized high incidence of vascular lesions/varices in association with these polypoid lesions.

Limitations include the retrospective design as well as the absence of polypectomy samples that would corroborate the presence of PHPs. However, this approach was considered unjustified in view of the EUS findings. Furthermore, categorization of the endoscopic findings was retrospective, but this was mandatory in order to achieve a more meaningful and uniform assessment of the endoscopy findings. In any case, the categorization was in complete agreement with the actual endoscopy reports.

\section{Conclusion}

Overall, this study demonstrated that there is a high incidence of varices in association with polypoid lesions in the context of $\mathrm{PH}$. Endoscopists should have a very high index of suspicion and be aware that relying on the endoscopic appearance alone can be deceiving. Attempting biopsies or polypectomies in this context should only be done if potential benefits outweigh the risks and further assessment using EUS should be considered to obtain more information regarding vascularity of such lesions. Use of image enhancing techniques to improve the diagnostic accuracy in the context of $\mathrm{PH}$ can potentially be of value, but requires further study.

\section{Competing interests}

None

References

[1] Spina GP, Arcidiacono R, Bosch J et al. Gastric endoscopic features in portal hypertension: final report of a consensus conference, Milan, Italy, September 19, 1992. J Hepatol 1994; 21: 461 - 467

[2] Kozarek RA, Botoman VA, Bredfeldt JE et al. Portal colopathy: prospective study of colonoscopy in patients with portal hypertension. Gastroenterology 1991; 101: 1192-1197

[3] De Palma GD, Rega M, Masone S et al. Mucosal abnormalities of the small bowel in patients with cirrhosis and portal hypertension: a capsule endoscopy study. Gastrointest Endosc 2005; 62: 529-534

[4] Devadason D, Murphy MS, Brown R et al. Duodenal capillary hemangiomatous polyps: a novel manifestation of extrahepatic portal hypertension? J Pediatr Gastroenterol Nutr 2007; 45: 114-116

[5] Lemmers A, Evrard S, Demetter P et al. Gastrointestinal polypoid lesions: a poorly known endoscopic feature of portal hypertension. United Eur Gastroenterol J 2014; 2: 189-196 
[6] Tang S-J, Jensen DM, Gralnek IM et al. Portal hypertensive enteropathy in a patient with polycystic liver disease: a unique endoscopic finding. Gastrointest Endosc 2002; 56: $924-926$

[7] Zeitoun J-D, Chryssostalis A, Terris B et al. Portal hypertensive duodenal polyp: a case report. World J Gastroenterol 2007; 13: 1451 1452

[8] Sawada K, Ohtake T, Ueno N et al. Multiple portal hypertensive polyps of the jejunum accompanied by anemia of unknown origin. Gastrointest Endosc 2011; 73: 179-182

[9] Boyd JT, Lee L. Portal hypertension-associated gastric polyps. BM] Case Rep 2014: doi:10.1136/bcr-2013-202312
[10] Lam MCW, Tha S, Owen D et al. Gastric polyps in patients with portal hypertension. Eur J Gastroenterol Hepatol 2011; 23: 1245-1249

[11] Lee TH, Jang JY, Jeong SW et al. Gastric polyposis associated with portal hypertension. Korean J Intern Med 2013; 28: 261

[12] Amarapurkar AD, Amarapurkar D, Choksi M et al. Portal hypertensive polyps: distinct entity. Indian J Gastroenterol 2013; 32: 195-199

[13] Panackel C, Joshy H, Sebastian B et al. Gastric antral polyps: a manifestation of portal hypertensive gastropathy. Indian J Gastroenterol 2013; 32: $206-207$

[14] Pai CG. Portal hypertensive polyp-what is in a name? Indian J Gastroenterol 2013; 32: $163-164$ 